\title{
Bilateral total deafness due to pontine haematoma
}

\author{
C A Egan, L Davies, G M Halmagyi
}

\begin{abstract}
A 64 year old woman with a predominantly midline pontine tegmental haemorrhage presented with bilateral total deafness. One week later reasonable pure-tone thresholds appeared but she still had total bilateral loss of speech discrimination. At that time contralateral acoustic reflexes were bilaterally absent, whereas ipsilateral acoustic reflexes and waves IV and $V$ of the brainstem auditory evoked potential were bilaterally preserved. It is proposed that this patient's hearing deficit was due to inactivation of the ventral acoustic striae decussating in the trapezoid body. This case supports the contention that in humans the ventral pontine acoustic decussation carries most of the neural signals required for hearing and perhaps all the neural signals required for speech perception.
\end{abstract}

(F Neurol Neurosurg Psychiatry 1996;61:628-631)

Keywords: deafness; speech perception; brainstem; trapezoid body; auditory evoked potentials

The auditory system decussates at several levels in the brainstem. There is no general agreement on the relative importance of the ipsilateral as opposed to the contralateral ascending auditory pathways in humans, in part because there are so few studies of hearing in patients with well localised brainstem lesions. Here we report a patient who temporarily became totally bilaterally deaf from a single pontine lesion which involved the trapezoid body.

\section{Neurology}

Department, Royal Prince Alfred Hospital, Sydney, Australia

CA Egan

Cavies

GM Halmagyi

Correspondence to: Dr GM Halmagyi, RPA Hospital, Camperdown, NSW 2050, Sydney, Australia.

Received 25 September 1995 and in revised form 29 April 1996

\section{Case report}

A 64 year old hypertensive woman collapsed without loss of consciousness after a brief episode of right sided paraesthesia and numbness. She was a native English speaker with no previous hearing problems. On examination she was fully conscious and alert but was totally deaf. She was able to lip read a little and so could give appropriate answers to simple spoken questions. She could read with comprehension. She had a left lateral gaze and a right abducens paresis. There was no facial weakness but there was a severe dysarthria characterised by loud slurred speech with an explosive quality; gag reflexes were absent bilaterally and tongue movements were full but slow. There was a profound loss of proprioception in both arms and in both legs but touch and pinprick sensation were normal. Limb movements were ataxic, probably due to the proprioceptive difficulties, but power was full. The tendon reflexes were brisk and both plantar responses were extensor.

Brain MRI disclosed an area of low signal on T2 weighted images in the dorsal pons, abutting the fourth ventricle, especially on the left (fig 1A). The T1 weighted images showed subtle signal attenuation in the same distribution. The changes were typical of haemorrhage. In addition, there was a small, old, lacunar infarct in the left lentiform nucleus. There was no cerebral cortical abnormality.

Over the next few days she recovered the ability to hear and to discriminate environmental sounds such as a telephone ringing, water running, footsteps, and a knock at the door, but remained unable to understand any spoken words.

Pure-tone audiometry showed a bilateral, moderately severe, sensorineural hearing loss (fig 2).

Speech audiometry showed that speech discrimination was bilaterally absent to both recorded and live voices even at $100 \mathrm{~dB}$. (The expected speech discrimination score if these pure-tone thresholds were due to cochlear lesions would be about $50 \%$ at $80 \mathrm{~dB}$.)

Acoustic reflex testing showed that ipsilateral acoustic reflexes (ARs) were present from each ear at normal thresholds at 500, 1000 and $2000 \mathrm{~Hz}$. Contralateral ARs were bilaterally absent.

Brainstem auditory evoked potentials (BAEPs) were elicited using $100 \mathrm{~dB}, 100 \mu$ s white noise rarefaction clicks at $12 \mathrm{~Hz}$. On stimulation of the right ear ipsilateral waves $\mathrm{I}$ to $\mathrm{V}$ were recorded at normal latencies (fig 3). On stimulation of the left ear ipsilateral waves II to $\mathrm{V}$ were recorded at normal latencies; wave V seemed to be reduced in amplitude and wave I was obscured by stimulus artefact.

After a month some speech comprehension had returned. One year later there was only a mild pure tone sensorineural hearing loss on the left with $100 \%$ speech discrimination at $50 \mathrm{~dB}$, and a mild to moderate pure tone hear- 


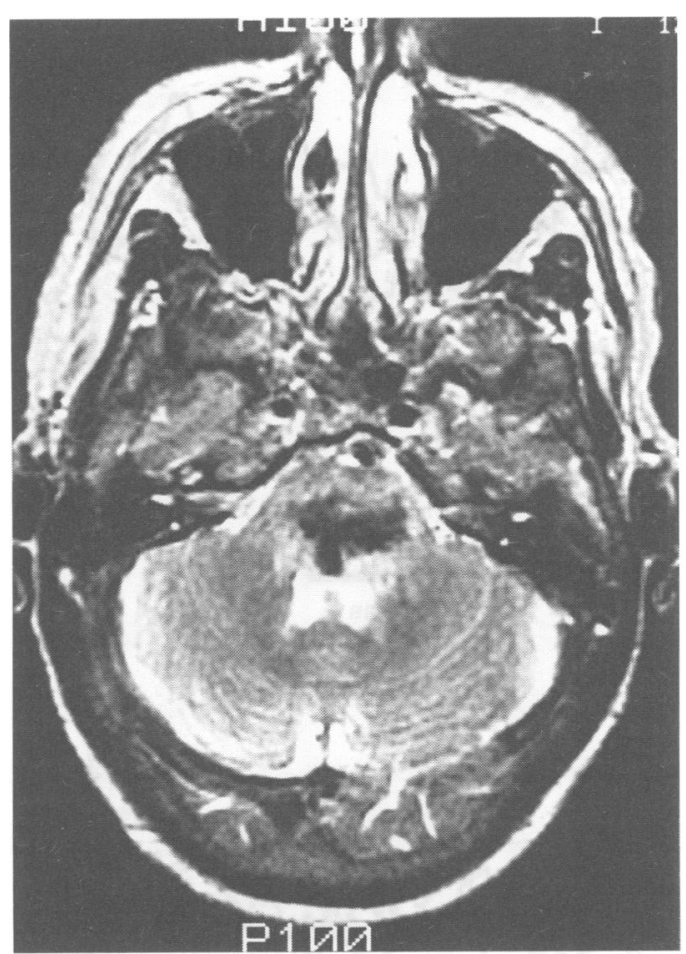

$A$

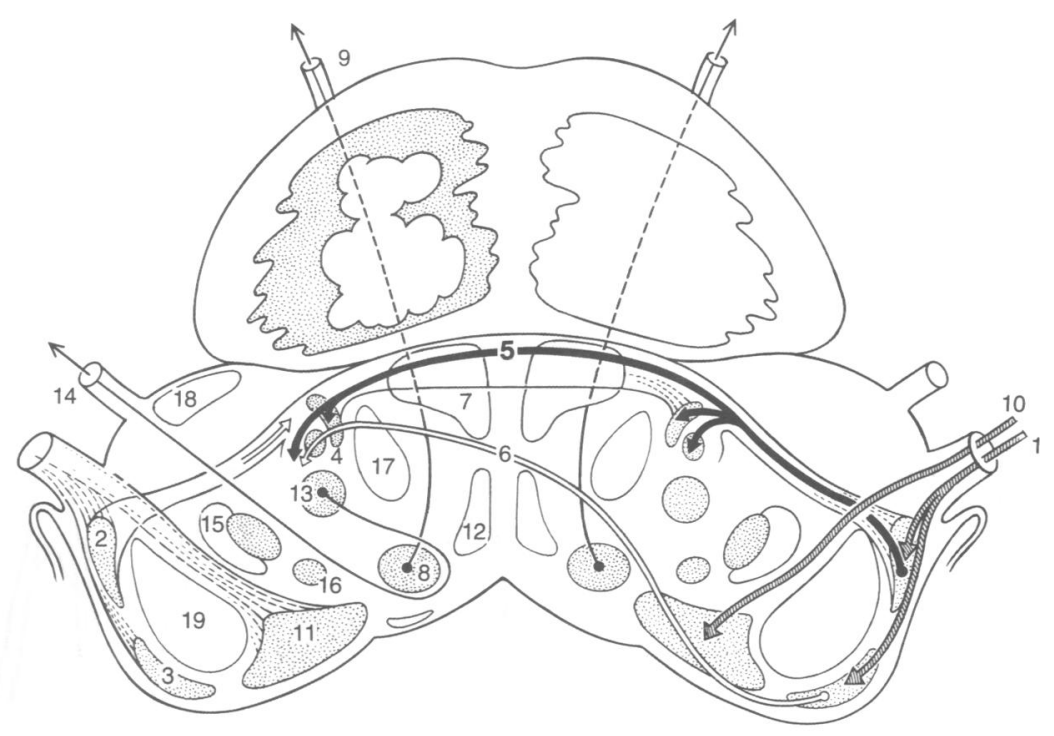

$B$

Figure 1 (A)T2 weighted axial MRI showing a recent midline haemorrhage in the pontine tegmentum at the level of the vestibulocochlear nerves. The lesion is well clear of the cochlear nuclei. (B) Schematic representation of brainstem structures at a level near the MRI shown in (A). From the clinical signs the main structures involved in the lesion apart from the trapezoid body (5) were: both medial lemnisci (7), both abducens fascicles (9) and the medial longitudinal fasciculus on the left (12). The cochlear nerves (1), the dorsal and ventral cochlear nuclei (2,3), the superior olivary nuclei (4), and the dorsal acoustic striae (6) were spared. Other structures shown in the figure, all spared, are the abducens nucleus (8); vestibular nerve (10); vestibular nucleus (11); facial nucleus (13); facial nerve (14); descending nucleus and tract of the trigeminal (15); tractus solitarius (16); central tegmental tract (17); anterior spinocerebellar tract (18), and inferior cerebellar peduncle (19). (Modified from Nieuwenhuys et al. The human central nervous system. New York:Springer Verlag, 1988.)

ing loss on the right with $85 \%$ speech discrimination at $70 \mathrm{~dB}$ (fig 2). The contralateral ARs had returned but were smaller in amplitude than the ipsilateral ARs. The other neurological deficits had also resolved except for a moderately severe left proprioceptive hemisensory
Right ear

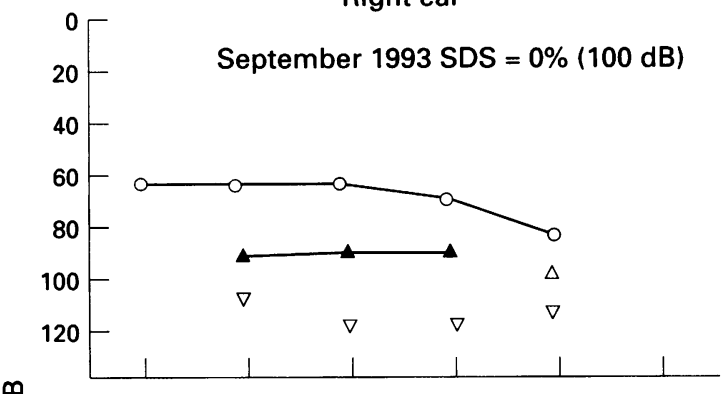

$\stackrel{9}{0}$

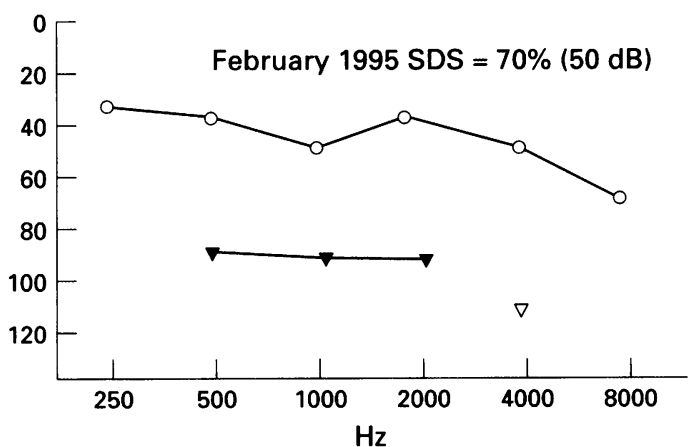

Left ear
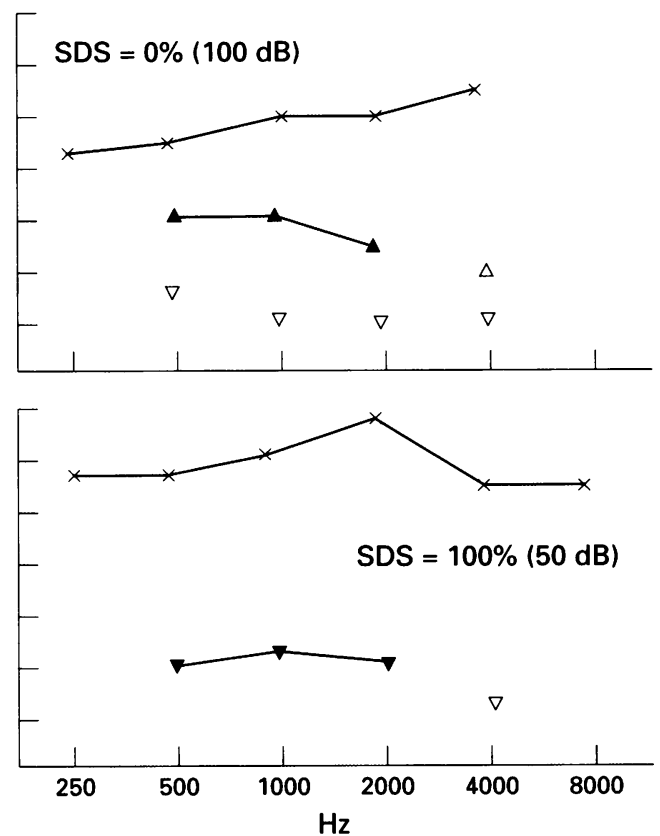

Figure 2 Pure tone audiograms, acoustic reflex thresholds, and speech discrimination scores (SDS) during the period of bilateral speech deafness (top row) and 18 months later when hearing was almost normal (bottom row). Note that when the patient had no speech discrimination from either ear, pure tone thresholds were reduced by only 30-70 dB; at that time ipsilateral acoustic reflexes were present at normal thresholds (A) whereas contralateral acoustic reflex could not be elicited with maximal stimulus intensity $(\nabla)$. (Note that at $4000 \mathrm{~Hz}$ the acoustic reflex can be absent in normal subjects.) Eighteen months later contralateral acoustic reflexes ( $)$ had returned. $O=$ right ear pure-tone threshold; $X=$ left ear pure-tone threshold; $\Delta=$ ipsilateral acoustic reflex threshold $(\Delta=$ absent reflex $) ; \nabla=$ contralateral acoustic reflex threshold $(\nabla=$ absent reflex). 
Figure 3 Brainstem auditory evoked potentials in response to $100 \mathrm{~dB}$ NHL, $100 \mu \mathrm{s}, 12 \mathrm{~Hz}$ white noise rarefaction clicks recorded on the same day as the audiogram on the top row of fig 2 . From top to bottom: right ear stimulate-left ear record $(R-L)$; right ear stimulate - right ear record $(R-R)$; left ear stimulateleft ear record $(L-L)$; left ear stimulate- right ear record $(L-R)$. On

stimulating the right ear, ipsilateral waves I to $V$ were recorded at normal latencies. On stimulating the left ear, ipsilateral waves II to $V$ were recorded at normal latencies; wave $V$ seemed to be reduced in amplitude and wave I was obscured by stimulus artefact.

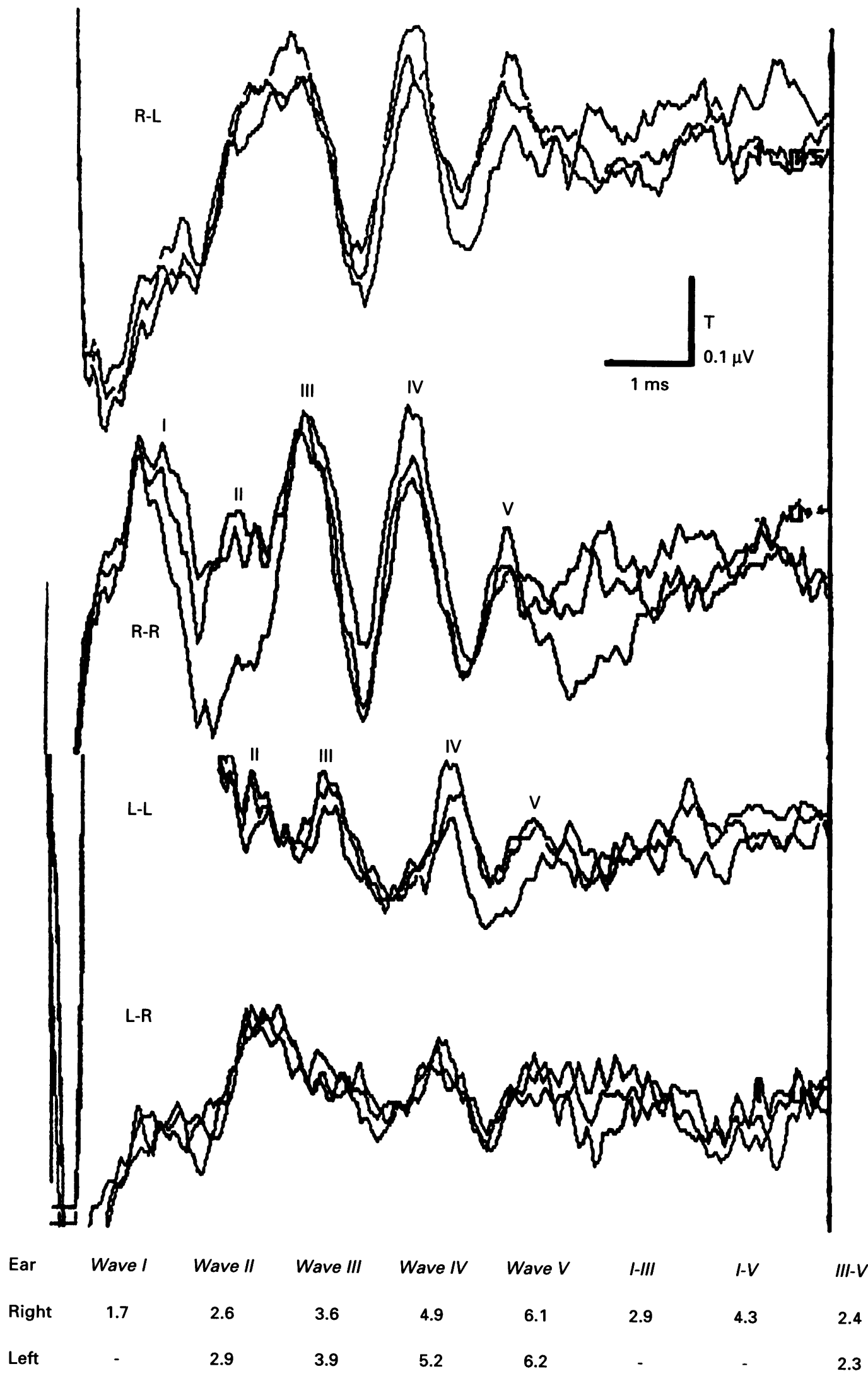

loss producing arm and leg sensory ataxia. Both plantar reflexes were still extensor.

\section{Discussion}

We could find only one previous case report of bilateral total or subtotal deafness due to a single pontine lesion. This was a patient who was locked-in and had no ARs at all, suggesting that the lesion was more extensive than in our patient and probably involved both superior olivary nuclei. ${ }^{1}$ Although bilateral selective impairment of speech discrimination with preservation of pure tone thresholds has been reported with brainstem lesions ${ }^{23}$ we could find no previous report of bilateral total loss of speech discrimination due to a single brainstem lesion. (In this discussion it is important 
to distinguish between bilateral total deafness followed by total loss of speech discrimination due to a single pontine lesion as reported here and the following: bilateral total deafness due to multiple pontine lesions ${ }^{4}$; bilateral total deafness due to midbrain lesions ${ }^{5}$; unilateral deafness due to a single pontine lesion ${ }^{6}$; and bilateral word deafness after bilateral auditory cortex lesions. ${ }^{7}$ )

The results could indicate that inactivation of the ventral acoustic striae can by itself produce bilateral total deafness or at least bilateral total loss of speech discrimination. This would suggest that in the human the trapezoid body transmits-via the contralateral lateral lemniscus-most of the neural signals required by more rostral auditory structures for hearing, and particularly for speech discrimination.

In cats, section of the trapezoid body produces not only an increase in pure tone thresholds but also a deficit in detection of salient sounds in a noisy environment ${ }^{8}$ - the type of deficit that would interfere with speech discrimination in a human; it also reduces the amplitudes of waves III to $\mathrm{V}$ of the BAEPs, but does not abolish them. ${ }^{9}$ By contrast, section of the other two pontine auditory commissures, the dorsal and the intermediate acoustic striae, produced no detectible deficits.

The evidence in our case that the trapezoid body was inactivated whereas the other pontine auditory structures were spared is persuasive. Bilateral loss of contralateral ARs with normal ipsilateral ARs can be best explained by a lesion of the trapezoid body. The first neuron of the AR is the primary cochlear afferent, which synapses in the ventral cochlear nucleus, and then projects to the ipsilateral superior olivary nucleus. From there the ipsilateral $A R$ pathway projects to stapedius motor neurons in and around the ipsilateral facial nucleus, whereas the contralateral AR pathway projects through the trapezoid body to the region of the contralateral facial nucleus. ${ }^{10}$

Furthermore, this patient's other neurological deficits, the pyramidal signs, the proprioceptive loss, and the ophthalmoplegia, all indicated involvement of structures which are either adjacent to the trapezoid body - the corticospinal tracts and the right medial longitudinal fasciculus - or intersect the trapezoid body-both medial lemnisci and both abducens fascicles. Structures at the same pontine level but distant from the trapezoid body-namely, the spinal trigeminal nuclei, the facial nuclei, the vestibular nuclei, the spinocerebellar tracts, the inferior cerebellar peduncles, and the central tegmental tractswere functionally spared. It seems then that not only was the trapezoid body inactivated by the lesion in this patient, but that it was the only auditory structure inactivated by the lesion (fig 1B).

The results also suggest that the ipsilateral lemniscal auditory pathway can by itself produce waves $I$ to $V$ of the BAEP. In cats, section of the trapezoid body attenuates but does not abolish the equivalent of waves IV and V of the surface recorded BAEP. ${ }^{9}$ Although there are no previous reports of BAEPs in humans with brainstem auditory lesions confined to the trapezoid body, there are data on BAEP from humans with unilateral lesions of the lateral lemniscus. These data are ambiguous; some indicate that the ipsilateral lemniscal pathway is necessary and sufficient to generate all components of the BAEP,, 112 whereas other data indicate that wave $\mathrm{V}$ exclusively arises from the contralateral lateral lemniscus. ${ }^{1314}$ In this patient, even though wave $\mathrm{V}$ might have been reduced in amplitude from one side, waves IV and V were still bilaterally present at the time that she had bilateral total loss of speech discrimination.

The assistance of GN Fuller, M Bray, and C Tsang is gratefully acknowledged.

1 Keane J. Locked-in syndrome with deafness. Neurology 1985;35:1395

2 Morales-Garcia C, Poole JP. Masked speech audiometry in central deafness. Acta Otolaryngol 1972;74:307-16.

3 Levine RA, Gardner JC, Fullerton BD, Stufflebeam SM, Furst M, Rosen BR. Muliple scleosis lesions of the auditory pons are not silent. Brain 1994;117:1127-41.

4 Davous $P$, Tillier J, Torrent J. Surdite totale apres infarctus protruberantiels multiples. Dolichoectasie du tronc basilaire. Rev Neurol (Paris) 1991;147:234-7.

5 Jani N, Laureno R, Mark A, Brewer C. Deafness after bilateral midbrain contusion: A correlation of magnetic resonance imaging with auditory brain stem evoked responses. Neurosurgery 1991;29:106-9.

6 Baratt HJ, Miller D, Rudge P. The site of the lesion causing deafness in multiple sclerosis. Scand Audiol 1988;17: deafness.

7 Coslett HB, Brashear HR, Heilman KM. Pure word deaf ness after bilateral primary auditory cortex infarcts. ness after bilateral primary

8 Masterton RB, Granger EM, Glendenning KK. The role of acoustic striae in hearing: mechanism for enhancement of sound detection in cats. Hear Res 1994;73:209-22.

9 Fullerton BC, Kiang NY. The effect of brainstem lesions on the brainstem auditory evoked potentials in the cat. Hear Res 1990;49:363-90.

10 Moller A. Auditory neurophysiology. f Clin Neurophysiol 1994;11:284-308.

11 Oh SJ, Kuba T, Soyer A, Choi S, Bonikowski FP, Vitek J. Lateralization of brainstem lesions by brainstem auditory potentials. Neurology 1981;31:14-8.

12 Hammond E, Wilder BJ, Goodman IJ, Hunter SB Auditory brain-stem potentials with unilateral pontine hemorrhage. Arch Neurol 1985;42:767-8.

13 Voordecker P, Brunko E, de Beyzl Z. Selective absence or attenuation of wave $\mathrm{V}$ of the brain-stem auditory evoked attenuation of wave $\mathrm{V}$ of the brain-stem auditory evoked potentials with in

14 Levine RA, Gardner JC, Fullerton BD, Stufflebeam SM, Carlisle EW, Furst M, Rosen BR, Kiang NYS. Effects of multiple sclerosis brainstem lesions on sound lateralization and brainstem auditory evoked potentials. Hear Res 93;68:73-88. 